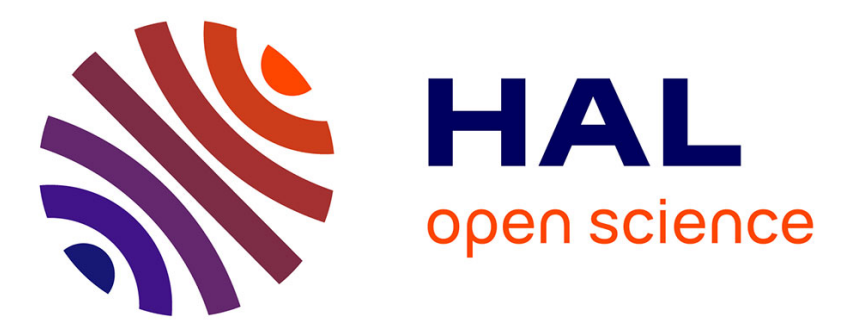

\title{
Détermination numérique de la distribution des concentrations ponctuelles au foyer des concentrateurs à facettes
}

R. Pasquetti

\section{- To cite this version:}

R. Pasquetti. Détermination numérique de la distribution des concentrations ponctuelles au foyer des concentrateurs à facettes. Revue de Physique Appliquée, 1984, 19 (6), pp.455-460. 10.1051/rphysap:01984001906045500 . jpa-00245217

\section{HAL Id: jpa-00245217 https://hal.science/jpa-00245217}

Submitted on 1 Jan 1984

HAL is a multi-disciplinary open access archive for the deposit and dissemination of scientific research documents, whether they are published or not. The documents may come from teaching and research institutions in France or abroad, or from public or private research centers.
L'archive ouverte pluridisciplinaire HAL, est destinée au dépôt et à la diffusion de documents scientifiques de niveau recherche, publiés ou non, émanant des établissements d'enseignement et de recherche français ou étrangers, des laboratoires publics ou privés. 


\title{
Détermination numérique de la distribution des concentrations ponctuelles au foyer des concentrateurs à facettes
}

\author{
R. Pasquetti \\ Département d'Héliophysique, Faculté de Saint Jérôme, 13397 Marseille Cedex 13, France
}

(Reçu le 28 novembre 1983, accepté le 15 mars 1984)

\begin{abstract}
Résumé. - Dans cette étude de détermination numérique de la distribution des concentrations ponctuelles au foyer de réflecteurs solaires, on décrit le support théorique à un programme de calcul applicable à des concentrateurs constitués de facettes de forme et de concavité quelconques, utilisant une réflexion du rayonnement incident. Le réflecteur est considéré comme une source de luminances et c'est par intégration sur sa surface des flux parvenant aux « points d'étude » que l'on détermine la concentration en ces points. La modélisation d'un miroir réel a été rendue possible car tous les paramètres définissant la facette et son positionnement peuvent être affectés d'erreurs de distribution aléatoire gaussienne. En dernier lieu est présenté un exemple d'application du modèle de calcul mis au point.
\end{abstract}

\begin{abstract}
This study, concerned with the numerical determination of local concentration distribution in the focal zone of solar reflectors, describes the theoretical basis of a computer program adapted to concentrators constituted with elementary mirrors of any shape and concavity, using one reflection of incident rays. The reflector is assimilated to an intensity source and the local concentration is calculated by integration on its surface of the flux reaching the " study point $"$. The modelization of a real concentrator is possible because all the characteristic parameters of the elementary mirror and its position can be altered with errors following Gaussian distributions. Lastly, the result of an application of this numerical program is given as an example.
\end{abstract}

\section{Introduction}

Dans la réalisation d'un capteur à concentration du rayonnement solaire, le plus délicat réside généralement dans la fabrication du réflecteur. En l'état actuel des techniques, l'obtention de surfaces réfléchissantes de grande superficie est en effet difficile et chère dès qu'un minimum de précision est requis. Pour des miroirs de grande dimension, la fragmentation est nécessaire (four solaire de Font Romeu [1], projet Pericles [2]). Le calcul des densités de flux, au foyer d'un "réflecteur fragmenté ", ne nécessite généralement pas, sauf, par exemple, effet d'interstice important, la prise en compte de la fragmentation du concentrateur.

Dans le cas des capteurs THEK [3-5], développés dans notre laboratoire, il s'agit de facettes triangulaires planes, accolées sur des supports paraboliques. La distribution des concentrations ponctuelles dans le volume focal est cette fois différente de celle qui serait obtenue avec un paraboloïde; elle dépend de la forme du support mais également de la forme (équilatérale plane) de la facette et de ses dimensions. La détermination précise des concentrations ponctuelles dans le volume focal d'un " réflecteur à facettes " nécessite un calcul au niveau de chacun des miroirs élémentaires, puis une sommation des résultats obtenus.
Le support théorique à un programme de calcul, mis au point pour l'étude des performances optiques des concentrateurs utilisant une réflexion du rayonnement solaire, est décrit dans le paragraphe 1. Le concentrateur et les facettes qui le couvrent peuvent être d'ouverture et de concavité quelconques. La modélisation d'un réflecteur réel a été rendue possible en engendrant, de manière aléatoire gaussienne, des approximations dans le positionnement des facettes et des erreurs dans leur réalisation. Il est tenu compte de la non-ponctualité de la source ainsi que de la nonuniformité de la distribution de sa luminance.

Dans le paragraphe 2 est présenté un exemple d'application du modèle mis au point : nous avons déterminé, pour diverses positions du soleil, la distribution des concentrations ponctuelles au foyer d'un capteur à concentration linéaire de l'énergie, dont le concentrateur est composé de facettes planes ou cylindriques.

\section{Le support théorique.}

1.1 Formulation INTÉGrale de la CONCENTRATION. - Eclairé par le soleil, le réflecteur se comporte luimême comme une source de rayonnement dont on va 
d'abord supposer connue la distribution des luminances; la théorie des transferts radiatifs permet alors de formuler l'éclairement sur une surface élémentaire.

Ainsi, soit (Fig. 1) $S$ une facette de point courant $\mathbf{P}$ et de vecteur unitaire normal $\mathbf{N}_{\mathbf{P}}$ et $\mathbf{N}_{\mathbf{A}}$ le vecteur

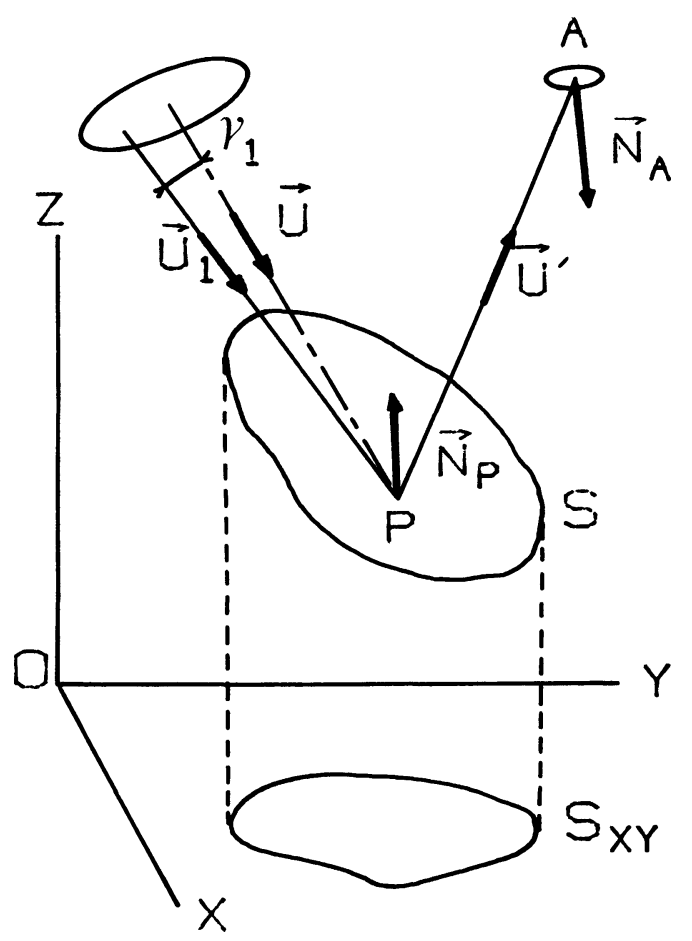

1. - Schéma de définition de la facette dans son repère.

[Definition schema of the mirror in its coordinate system.]

unitaire normal à la surface élémentaire d'étude centré en A. L'éclairement $\phi$ en A s'exprime sous la forme d'une intégrale dans l'angle solide $\Omega$ sous lequel le point A voit $S$. Avec $L_{\mathrm{P}, \mathrm{A}}$ la luminance en $\mathrm{P}$ vers A et $\mathbf{u}^{\prime}$ le vecteur unitaire du segment de droite PA :

$$
\phi=\int_{\Omega}-L_{\mathrm{P}, \mathrm{A}} \underbrace{\mathbf{u}^{\prime} \cdot \mathbf{N}_{\mathrm{A}}}_{\leqslant 0} \mathrm{~d} \Omega .
$$

Une telle intégrale peut se ramener à une intégrale double dans un domaine plan.

Dans le repère $O x y z$ de vecteurs unitaires $\mathbf{i}, \mathbf{j}, \mathbf{k}$, avec $\mathrm{d} S_{x y}$ la projection de la surface élémentaire $\mathrm{d} S$ dans le plan $x y$ et sachant que :

$$
\begin{aligned}
\mathrm{d} \Omega & =\frac{\mathrm{d} S\left(\mathbf{N}_{\mathrm{p}} \cdot \mathbf{u}^{\prime}\right)}{\mathrm{PA}^{2}} \\
\mathrm{~d} S_{x y} & =\mathrm{d} S \mathbf{N}_{\mathrm{p}} \cdot \mathbf{k}
\end{aligned}
$$

(en $\left.\operatorname{supposant}\left(\mathbf{N}_{\mathrm{P}}, \mathbf{k}\right)>0\right)$

$$
\mathbf{N}_{\mathbf{P}}=\nabla S /|\nabla S|
$$

( $\left.{ }^{1}\right) \nabla$ : opérateur hamiltonien. il vient :

$$
\mathrm{d} \Omega=\frac{\nabla S \cdot \mathbf{u}^{\prime}}{\mathrm{PA}^{2}} \cdot \frac{1}{\nabla S \cdot \mathbf{k}} \mathrm{d} S_{x y}
$$

ou, plus simplement, si l'on suppose que $S$ peut s'exprimer sous forme explicite en $z$ :

$$
\mathrm{d} \Omega=-\frac{(p \mathbf{i}+q \mathbf{j}-\mathbf{k}) \mathbf{u}^{\prime}}{\mathrm{PA}^{2}} \mathrm{~d} S_{x y}
$$

avec $p=\frac{\partial z}{\partial x}$ et $q=\frac{\partial z}{\partial y}$.

Dans (1) il faut maintenant exprimer la distribution des luminances $L_{\mathbf{P}, \mathbf{A}}$ en fonction de celle $L_{\gamma}$ du soleil. $L_{\gamma}$ est symétrique de révolution et non nulle si l'angle $\gamma$ est compris entre 0 et $\gamma_{\mathbf{s}}$, le demi-angle d'ouverture du soleil.

Soit $\mathbf{u}_{1}$ le vecteur unitaire qui, après réflexion en $P$, se trouve en $u^{\prime}$. Les lois de la réflexion spéculaire conduisent à l'expression vectorielle suivante :

$$
\mathbf{u}_{1}=\mathbf{u}^{\prime}-2 \mathbf{N}_{\mathbf{P}}\left(\mathbf{u}^{\prime} \cdot \mathbf{N}_{\mathbf{p}}\right)
$$

Si u est le vecteur unitaire issu du centre du disque solaire et orienté vers $\mathbf{P}$, on a, au facteur de transmission de l'atmosphère près, et avec $\gamma_{1}=\left(\mathbf{u}, \mathbf{u}_{1}\right)$ :

$$
L_{\mathrm{P}, \mathbf{A}}=\rho L_{\gamma_{1}}
$$

$\rho$ etant e acteur e re exion, suppose omni : e tionnel, du miroir.

La concentration $C$ en $\left(A, N_{A}\right)$ est égale au rappor de l'éclairement $\phi$ sur la surface élémentaire d'étude à celui, $H$, reçu au sol perpendiculairement au rayonnement solaire incident. Or, au facteur de transmission de l'atmosphère près :

$$
H=\pi \gamma_{\mathrm{s}}^{2} \bar{L}_{\gamma}
$$

(avec $\bar{L}_{\gamma}$ la valeur moyenne de $L_{\gamma}$ sur le disque solaire).

Finalement, d'après $(1),(6),(8)$ et $(9)$, et en posant :

$$
g\left(\gamma / \gamma_{\mathrm{S}}\right)=L_{\gamma} / \bar{L}_{\gamma}
$$

il vient :

$$
C=\frac{\rho}{\pi \gamma_{\mathbf{S}}^{2}} \int_{\mathbf{S}_{x y}} g\left(\frac{\gamma}{\gamma_{\mathrm{S}}}\right) \frac{(p \mathbf{i}+q \mathbf{j}-\mathbf{k}) \mathbf{u}^{\prime}}{\mathbf{P A}^{2}} \mathbf{u}^{\prime} \cdot \mathbf{N}_{\mathrm{A}} \mathrm{d} S_{x y} .
$$

Si l'on ne tient pas compte de la distribution des luminances sur le disque solaire, $g\left(\gamma / \gamma_{\mathrm{s}}\right)=1$. Dans le cas contraire, il existe plusieurs formulations, par exemple, celle utilisée en (6) :

$$
g\left(\frac{\gamma}{\gamma_{\mathrm{s}}}\right) \cong 1,207\left(1-0,5138\left(\frac{\gamma}{\gamma_{\mathrm{s}}}\right)^{4}\right)
$$


Comme en $[7,8]$, nous avons retenu l'expression proposée par P. D. José [9] :

$$
g\left(\frac{\gamma}{\gamma_{\mathrm{s}}}\right)=1,255 \frac{1+1,5641 \sqrt{1-\left(\frac{\gamma}{\gamma_{\mathrm{s}}}\right)^{2}}}{2,5641} .
$$

\subsection{DÉTERMINATION DE LA CONCENTRATION POUR UN} ENSEMBLE DE FACETTES. - L'expression (11), qui ne restreint pas la généralité de notre démarche, présente une forme bien transcriptible du point de vue informatique : il s'agit, à un facteur de proportionalité près, de l'intégrale numérique sur un domaine plan, a priori quelconque, d'une fonction $f(x, y)$ dépendante de grandeurs elles-mêmes fonction de point sur le domaine d'intégration.

Cinq fonctions et un ensemble de paramètres $F$ servent à la définition effective de la facette dans un repère, dont le choix n'est soumis qu'à la condition admise en (3); les trois premières permettent une caractérisation de la concavité et les autres celles de l'ouverture :

$$
\left.\begin{array}{l}
-z_{\mathrm{p}}(x, y, F) \\
-p_{\mathrm{p}}(x, y, F) \\
-q_{\mathrm{p}}(x, y, F) \\
-\max (y)(x, F) \\
-\min (y)(x, F)
\end{array}\right\}
$$

Les deux dernières de ces fonctions définissent $S_{x y}$. $\mathrm{Si}$ les coordonnées polaires sont préférables, on pourra leur substituer une formulation équivalente en $(r, \theta)$.

Pour un ensemble de facettes, il est nécessaire de sommer les résultats obtenus en $\left(A, \mathbf{N}_{A}\right)$. Pour suivre la démarche ci-dessus définie, il suffit d'effectuer chacun des calculs élémentaires dans le repère propre à chacune des facettes. On supposera ces repères choisis de telle manière que la caractérisation de ces facettes puisse s'effectuer d'une manière sinon identique (si les facettes ne le sont pas), du moins similaire, une telle hypothèse étant d'ailleurs peu restrictive du point de vue informatique. Ainsi, seul l'ensemble des paramètres $F$ sera considéré propre à chaque facette.

Avec $(\mathrm{O}, \mathbf{i}, \mathbf{j}, \mathbf{k})_{1}$ le repère de la facette indicée 1 et $(\mathrm{O}, \mathbf{i}, \mathbf{j}, \mathbf{k})$ le repère du réflecteur, dans le programme numérique mis au point, les coordonnées du point origine $\mathrm{O}_{1}$ et les angles $\left(\mathbf{i}, \operatorname{proj}_{x y} \mathbf{i}_{1}\right),\left(\mathbf{i}, \operatorname{proj}_{x y} \mathbf{k}_{1}\right)$ et $\left(\mathbf{k}, \mathbf{k}_{1}\right)$ servent au positionnement des facettes sur le concentrateur $\left({ }^{3}\right)$.

1.3 PRISE EN COMPTE DES TOLÉRANCES DE FABRICATION. - En héliotechnique, les contraintes économiques sont telles que les réflecteurs ne peuvent pré-

$\left({ }^{2}\right) \max , \min :$ valeurs maximales et minimales de.

$\left({ }^{3}\right) \operatorname{proj}_{x y}$ : projection de, sur le plan Oxy. tendre à la qualité des appareils d'optique traditionnelle. Il est donc impératif, dans les calculs des concentrateurs à facettes, de tenir compte des tolérances de fabrication de ces facettes et de la qualité optique des miroirs utilisés.

La prise en compte de la qualité optique du miroir peut, en première approximation, s'effectuer en majorant l'angle d'ouverture du soleil $2 \gamma_{\mathrm{s}}$, alors qu'un calcul exact nécessiterait la connaissance des indicatrices de réflexion (fonction de l'angle d'incidence). Plus commodément, on caractérise habituellement la dispersion d'un miroir en supposant qu'indépendamment de l'angle d'incidence, le facteur de réflexion directionnel $\rho_{\Delta r}$ est une fonction de l'angle $\Delta r$, égal à la déviation du faisceau réfléchi par rapport à sa direction spéculaire. Dans cette hypothèse, on peut envisager de substituer à la distribution des luminances $g\left(\gamma / \gamma_{\mathrm{s}}\right) \bar{L}_{\gamma}$ sur le disque solaire une distribution fictive $g^{\prime}\left(\gamma / \gamma_{\mathrm{s}}\right) \bar{L}_{\gamma}$ traduisant les phénomènes de dispersion. Il a été montré [10] que, dans les cas les plus simples, on avait une distribution des $\rho_{\Delta r}$ de type gaussien.

La facette est, en général, une figure géométrique simple dont on peut faire apparaître les grandeurs caractéristiques dans l'ensemble $F_{1}$ des valeurs intervenant dans la définition de la facette indicée 1 . La prise en compte des tolérances de fabrication peut donc s'effectuer en substituant à l'ensemble $F_{1}$ un ensemble $F_{1}^{\prime}$ contenant les valeurs initiales affectées d'erreurs engendrées aléatoirement à l'intérieur des tolérances. Nous avons opté pour une loi de distribution gaussienne de ces erreurs. L'ensemble $F_{1}^{\prime}$ est engendré d'après $F_{1}$ et deux autres ensembles de valeurs contenant, d'une part, les valeurs moyennes des erreurs et, d'autre part, les écarts-types correspondants. Pratiquement, les erreurs moyennes sont en général prises nulles et les écarts-types égaux à la moitié de la tolérance, Avec $f$ un paramètre de $F_{1}, \bar{e}_{\mathrm{f}}$ la valeur moyenne de l'erreur et $\sigma_{\mathrm{f}}$ l'écart-type, $f^{\prime}$ de $F_{1}^{\prime}$ est calculé suivant une expression approximée [11] d'après :

$$
f^{\prime}=f+\bar{e}_{\mathrm{f}}+\left(\sum_{m=n}^{m=n+12} W_{m}-6\right) \sigma_{\mathrm{f}}
$$

où les $W_{m}$ sont des nombres pseudo-aléatoires entre 0 et 1 .

Concernant le positionnement des facettes, nous avons rendu possible la prise en compte de défauts d'orientation en inclinant de manière aléatoire gaussienne les repères de chacune des facettes. Ainsi, au repère $(O, \mathbf{i}, \mathbf{j}, \mathbf{k})_{1}$ on substitue un repère $\left(O, \mathbf{i}^{\prime}, \mathbf{j}^{\prime}, \mathbf{k}^{\prime}\right)_{1}$ tel que l'angle $\left(\mathbf{k}_{1}, \mathbf{k}_{1}^{\prime}\right)$ soit engendré aléatoirement dans un plan de rotation défini, ou lui-même engendré de manière purement aléatoire. Fixer le plan de rotation est intéressant pour les capteurs à concentration linéaire de l'énergie; dans le cas général, avec $\theta$ un angle aléatoire entre $O$ et $\pi$ définissant ce plan et $e$ l'angle d'erreur : 


$$
\left[\begin{array}{l}
\mathbf{i}_{1}^{\prime} \\
\mathbf{j}_{1}^{\prime} \\
\mathbf{k}_{1}^{\prime}
\end{array}\right]=\left[\begin{array}{lcc}
\cos ^{2} \theta+\sin ^{2} \theta \cos e & \cos \theta \sin \theta(1-\cos e) & -\sin \theta \sin e \\
\sin \theta \cos \theta(1-\cos e) & \sin ^{2} \theta+\cos ^{2} \theta \cos e & \cos \theta \sin e \\
\sin e \sin \theta & -\sin e \cos \theta & \cos e
\end{array}\right]\left[\begin{array}{l}
\mathbf{i}_{1} \\
\mathbf{j}_{1} \\
\mathbf{k}_{1}
\end{array}\right]
$$

où $\theta=\left(\mathbf{i}_{1}, \mathbf{k}_{1} \wedge \mathbf{k}_{1}^{\prime}\right)(\operatorname{Mod} \pi)\left({ }^{4}\right)$.

Le repère $\left(O, i^{\prime}, j^{\prime}, \mathbf{k}^{\prime}\right)_{1}$ étant défini, on exprime dans ce repère le vecteur $u$, les coordonnées de chacun des points d'étude $\mathrm{A}$ et les composantes des vecteurs $\mathbf{N}_{\mathbf{A}}$.

L'intégrale (11) est ensuite calculée numériquement d'après une méthode de Simpson à pas variable permettant un contrôle de la précision demandée au calcul.

\section{Un exemple d'application.}

Ce modèle de calcul nous a d'abord permis une étude détaillée des facettes habituellement utilisées en Energie Solaire : sphérique, cylindrique, tronconique et plane [12]. Les résultats présentés ci-dessous sont relatifs à un capteur dont un prototype, dénommé "structure rigide à lames orientables ", a été développé et réalisé au Département d'Héliophysique dans le cadre d'une convention de recherche [13]. Il s'agit d'un capteur à concentration linéaire de l'énergie, orienté Est-Ouest, dont le concentrateur est constitué d'un ensemble de dix lames mobiles $\mathrm{r}$ axe et de lar eur $D$ é ale à $0,15 \mathrm{~m}$; ces axes, horizontaux et distants de $0,2 \mathrm{~m}$, se situent dans un plan incliné de $10^{\circ}$. La chaudière inclinée à $25^{\circ}$ est quasiment au sol; son axe est parallèle aux lames et situé à $2 \mathrm{~m}$ en avant du réflecteur.

Le réflecteur est actuellement équipé de lames planes; nous avons essayé de mettre en évidence l'intérêt de l'utilisation de lames légèrement concaves. A cet effet, nous avons utilisé le modèle dont le support théorique a été décrit au paragraphe 1, en considérant des facettes cylindriques de rayon fini et infini.

La difficulté rencontrée ici, qui n'est d'ailleurs pas spécifique au capteur étudié, réside dans la possibilité d'effet d'ombre interfacettes, à l'incidence comme à l'émergence. Pour contourner cette difficulté, il suffit, préalablement à la détermination des concentrations ponctuelles, de déterminer, pour la position envisagée du soleil, la surface efficace de chacune des facettes. Dans le cas du capteur étudié, le calcul de la largeur efficace est élémentaire en raisonnant dans le plan méridien et sachant que les distances interfacettes sont négligeables par rapport à leur rayon.

Figures 2 et 3 sont présentées les résultats obtenus, c'est-à-dire pour diverses directions du soleil caractérisées par les angles d'azimut et de hauteur $a$ et $h$, la distribution des concentrations ponctuelles au

(4) Mod : modulo.

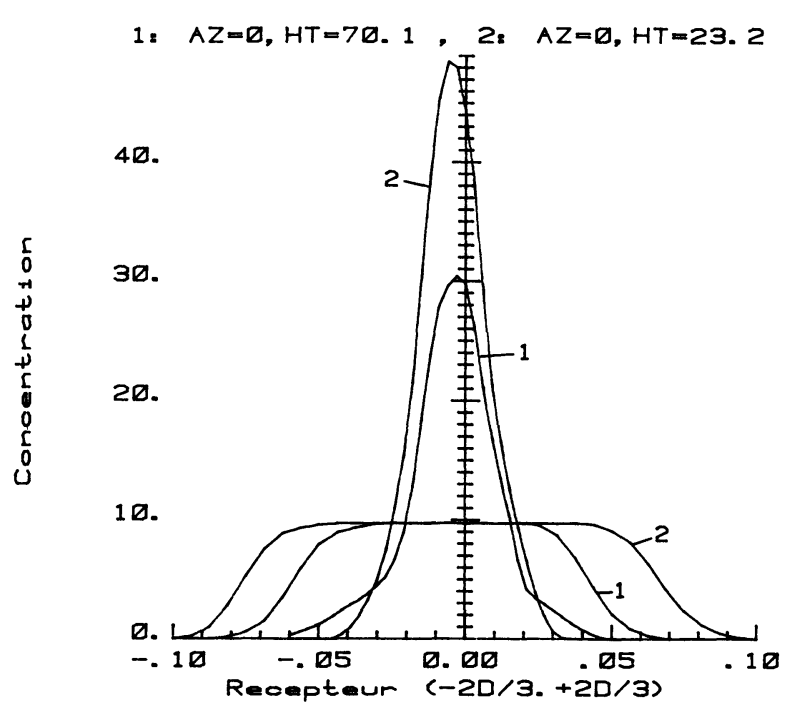

Fig. 2. - Concentrations au foyer de réflecteurs à lames cylindriques et planes pour deux angles de hauteur du soleil.

[Local concentrations on the focal area of reflectors constituted with cylindrical or flat mirrors for two solar altitude angles.]

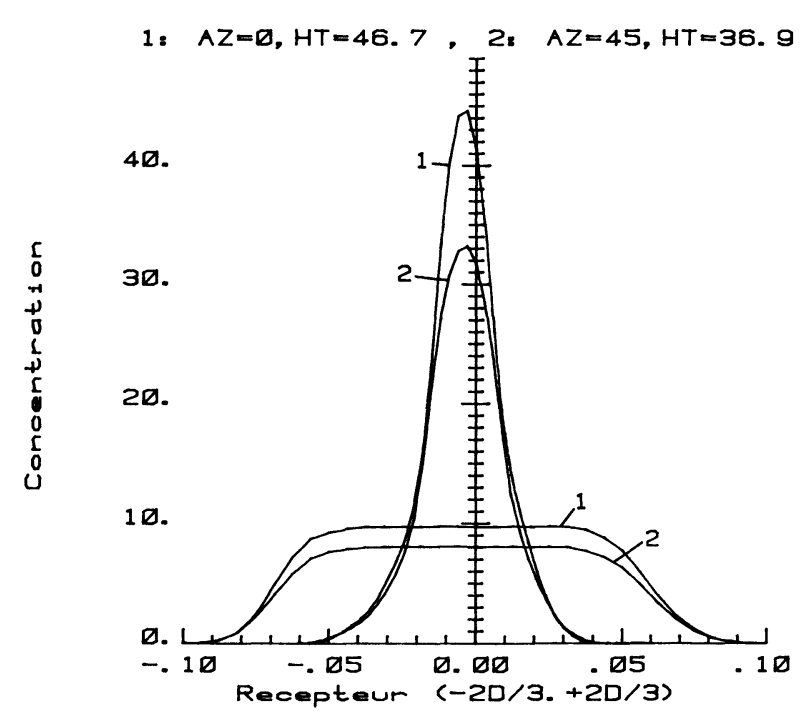

Fig. 3. - Concentrations au foyer de réflecteurs à lames cylindriques et planes pour deux angles d'azimut du soleil.

[Local concentrations on the focal area of reflectors constituted with cylindrical or flat mirrors for two solar azimuth angles.]

foyer, entre $-2 D / 3$ et $+2 D / 3$, pour des concentrateurs constitués de facettes planes et cylindriques. On a considéré un demi-angle d'ouverture du soleil 
de $18^{\prime}$ pour tenir compte de la qualité optique du miroir. La tolérance de réalisation de la facette a porté sur son rayon $(5 \mathrm{~m} \pm 0,5 \mathrm{~m})$ et la tolérance angulaire sur les normales aux facettes a été prise égale à $\pm 0,25^{\circ}$.

A Marseille et au midi solaire :

Figure 2 : Solstice d'été $\left(a=0^{\circ}, h=70,1^{\circ}\right)$ Solstice d'hiver $\left(a=0^{\circ}, h=23,2^{\circ}\right)$.

Figure 3 : Equinoxes $\left(a=0^{\circ}, h=46,7^{\circ}\right)$ Equinoxes $\left(a=45^{\circ}, h=36,9^{\circ}\right)$ à $14 \mathrm{~h} 18^{\prime}$.

L'utilisation de lames cylindriques permet donc de doubler, approximativement, la concentration géométrique mais entraîne l'apparition de concentrations ponctuelles maximales sensiblement supérieures à celles obtenues avec des lames planes. On remarque également, de manière qualitative, que la distribution des concentrations ponctuelles correspondant aux lames planes présente, en fonction de la position du soleil, une meilleure "stabilité ". Une telle constatation peut être étendue aux défauts de pointage et pour le mettre en évidence, nous avons initialisé différemment le générateur de nombres aléatoires. On peut ainsi comparer, figure 4 , un des résultats obtenus (courbe en pointillés) avec celui précédemment présenté pour le solstice d'été. Bien que peu représentatif, car trois angles d'inclinaison sur dix excèdent ici la valeur de deux écarts-types, ce dernier résultat ne doit pas surprendre et n'est pas dénué de signification physique, compte tenu du faible nombre de facettes et de la tolérance angulaire relativement importante qui a été retenue.

\section{Conclusion.}

La fabrication répétitive des facettes devant constituer à terme une solution économique, il est important de s'intéresser aux réflecteurs pouvant résulter de leur assemblage. Dans cette optique, le modèle que nous

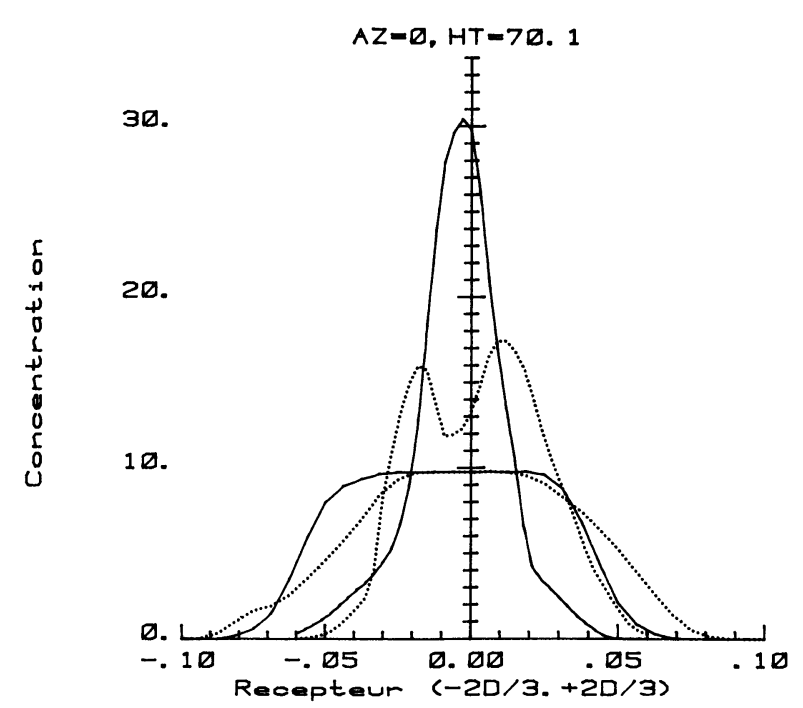

Fig. 4. - Concentrations au foyer de réflecteurs à lames cylindriques et planes pour deux distributions des angles de pointage.

[Local concentrations on the focal area of reflectors constituted with cylindrical or flat mirrors for two tracking angle distributions].

avons mis au point constitue un outil permettant leur caractérisation théorique mais également l'étude de l'incidence des imprécisions de réalisation par simulation de miroirs réels.

Par rapport à divers travaux traitant de problèmes similaires (ainsi [14]), notre approche apparaît comme d'une grande généralité puisque non spécifique à un réflecteur particulier; en contrepartie, et notamment si on la compare aux algorithmes qui ont pu être élaborés pour l'étude des champs d'héliostats (ainsi [15-18]), elle est certainement plus lourde, mais peut toutefois prétendre à une meilleure précision.

Bibliographie

[1] Essais de la centrale électrosolaire de 1 MWth (Rapport final). Laboratoire d'Energétique Solaire, C.N.R.S.

[2] AuthieR, B., Réflexions sur les collecteurs solaires à températures élevées $\left(260^{\circ} \mathrm{C}-1000^{\circ} \mathrm{C}\right)$. Thèse de Doctorat d'Etat, Marseille, janvier 1982.

[3] Peri, G. et al., Solar thermal electric power in the range of medium size units : the French program THEK. Congrès ISES, Atlanta (U.S.A.), 28 mailer juin 1979.

[4] Desautel, J., Le capteur héliothermique pseudoparabolique à facettes réfléchissantes planes THEK : l'Expérience THEK 1 . Thèse de Docteur Ingénieur, Marseille, juillet 1983.

[5] AudiberT, M., Programme solaire THEK : production de chaleur à des fins industrielles. Rev. Gén. Therm. 234235 (1981) 509-518.
[6] Hennet, J. C., Méthodologie d'évaluation et de conception de champs d'héliostats focalisants. Thèse de Doctorat d'Etat, Toulouse, mai 1982.

[7] Evans, D. L., On the performance of cylindrical parabolic solar concentrators with flat absorbers. Solar Energy 19 (1977) 379-385.

[8] Ya. Umarov, G., Zakhidov, R. A., Khokzhaev, A. Sh., Radiant vector distribution in the radiant field of a parabolocylindric concentrator. Geliotecknika, 12, 1 (1976) 27-32.

[9] JosE, P. D., The flux through the focal spot of a solar furnace. Solar Energy, 1 (1957) 4.

[10] Butler, B. L., Pettit, R. B., Optical evaluation techniques for reflecting solar concentrators. SPIE, 114, Optics applied to solar energy conversion, 1977, 43-49. 
[11] HAMming, R. W., Numerical methods for scientists and engineers (McGraw-Hill, New York) 1962, p. 34389.

[12] PASQuetTi, R., La conversion héliothermique à moyenne température; Sélectivité et concentration; Application aux capteurs plans et aux concentrateurs à facettes. Thèse de Doctorat d'Etat, Marseille, mars 1983.

[13] Convention de Recherche $\mathrm{n}^{\circ} 81.1013$ TCFD/GERES. Etude d'un capteur-convertisseur comportant Structure Rigide à Lames Orientables (S.R.L.O.). Rapport final, avril 1983.

[14] ZaKhidov, R. A., KhodzhaEv, S. H., Energy computation of concentrating capability of paraboloïdal facets. Gelioteknika 12, 5 (1976) 26-30.
[15] Courrege, Ph., Calcul des flux radiatifs d'une centrale solaire à concentration : (1) Eléments de la théorie du transfert radiatif. Rapport THEM 78.14, 20 septembre 1976.

[16] Mersier, C., Les centrales électro-solaires à tour. Optimisation du champ de réflecteurs et application à l'effet de taille du champ. Revue Phys. Appl. 14, $\mathrm{n}^{\circ} 1$ (1979) 1.

[17] Gentili, B., Une méthode d'implantation et de calcul des performances du champ de miroirs d'une centrale solaire à tour. GESER 001, novembre 1979.

[18] Chaput, E., Optimisation d'héliostats focalisants à surface discontinue. Thèse de Troisième Cycle, Poitiers, juillet 1983. 